\title{
A single session of submaximal grip strength training with or without high-definition anodal-TDCS produces no cross-education of maximal force
}

\section{RAZIE J. ALIBAZI ${ }^{1}$ | ASHLYN K. FRAZER ${ }^{1}$ | JAMIE TALLENT² | ALAN J. PEARCE ${ }^{3}$ | TIBOR HORTOBÁGYI ${ }^{4}$ | DAWSON KIDGELL ${ }^{1}$}

1 Department of Physiotherapy, School of Primary and Allied Health Care, Faculty of Medicine, Nursing and Health Science, Monash University, Melbourne, Australia.

2 Faculty of Sport, Health and Applied Sciences, St Mary's University, Twickenham, England.

${ }^{3}$ College of Science, Health and Engineering, La Trobe University, Melbourne, Australia.

${ }^{4}$ Center for Human Movement Sciences, University of Groningen, University Medical Center Groningen, Groningen, The Netherlands.

Correspondence to: Dawson J Kidgell, PhD. Department of Physiotherapy, School of Primary and Allied Health Care, Faculty of Medicine, Nursing and Health Science, Monash University, PO Box 527 Frankston, Victoria, Australia, 3199.

email: dawson.kidgell@monash.edu

https://doi.org/10.20338/bjmb.v15i3.223

\begin{tabular}{|c|c|}
\hline \multicolumn{2}{|c|}{$\begin{array}{l}\text { - The effects of bi-hemispheric a-tDCS on } \\
\text { modulating corticospinal excitability and motor } \\
\text { function were studied. } \\
\text { - A single-session of submaximal grip training } \\
\text { with or without high-definition bi-hemispheric a- } \\
\text { tDCS did not influence maximal grip force. } \\
\text { - Bi-hemispheric a-tDCS produces does not } \\
\text { improve maximal grip force nor does it affect } \\
\text { the excitability of the M1. }\end{array}$} \\
\hline \multicolumn{2}{|c|}{ ABBREVIATIONS } \\
\hline AMT & Active motor threshold \\
\hline AURC & Area under the recruitment curve \\
\hline CS & Conditioning stimulus \\
\hline ECRB & Extensor carpi radialis brevis \\
\hline FCR & Flexor carpi radialis \\
\hline ICF & Intracortical facilitation \\
\hline ISIs & Interstimulus intervals \\
\hline $\mathrm{LICl}$ & Long-intracortical inhibition \\
\hline M1 & Motor cortex \\
\hline MEP & Motor-evoked potential \\
\hline$M_{\text {MAX }}$ & $\begin{array}{l}\text { No further increase observed in } \\
\text { the amplitude of the sEMG } \\
\text { response }\end{array}$ \\
\hline MVIC & $\begin{array}{l}\text { Maximum voluntary isometric } \\
\text { contraction }\end{array}$ \\
\hline$\eta_{p^{2}}^{2}$ & Partial eta-squared \\
\hline rms & Root-mean-square \\
\hline sEMG & Surface electromyography \\
\hline $\mathrm{SICl}$ & $\begin{array}{l}\text { Short-interval intracortical } \\
\text { inhibition }\end{array}$ \\
\hline ST & Strength training \\
\hline TCDS & $\begin{array}{l}\text { Transcranial direct current } \\
\text { stimulation }\end{array}$ \\
\hline TMS & Transcranial Magnetic Stimulation \\
\hline TS & Test stimulus \\
\hline
\end{tabular}

PUBLICATION DATA

Received 17122020

Accepted 09042021

Published 01092021
BACKGROUND: Previous studies suggest that cross-education of strength may be modulated by increased corticospinal excitability of the ipsilateral primary motor cortex (M1) due to cross-activation. Conventional anodal-transcranial direct current stimulation (TDCS) has been shown to acutely increase bilateral cortical excitability and impart a cross-education effect. However, no study has examined the influence of bilateral TDCS of both M1 (i.e., ipsi- and contralateral M1) and how it affects corticospinal excitability, cross-activation and cross-education of muscle strength.

METHOD: Twelve participants (8 women and 4 men; aged 18-50 years) underwent three conditions in a randomized crossover design: (1) submaximal grip training and single-site unilateral-high definition-TDCS (ipsilateral to the training side) (2) submaximal grip training and bilateral anodal-high definition-TDCS, and (3) submaximal grip training and sham-high definition-TDCS. Submaximal gripping task involved a single-session of unilateral training which was squeezing the transducer at $70 \%$ of maximum voluntary isometric contraction (MVIC) grip force and performing four sets of 10 isometric contractions held steadily for 5 seconds. Anodal-high definition-TDCS was applied for $15 \mathrm{~min}$ at $1.5 \mathrm{~mA}$ over right M1 (unilateral single-site) or left and right M1s (bilateral), and in a sham condition during submaximal grip training. Participants were pseudorandomized to receive either single-site or bilateral $\mathrm{M} 1$ stimulation with each session separated by one-week. Before and after each session, MVIC force of ipsilateral and contralateral gripping, ipsilateral stimulus-response curve, shortinterval intracortical inhibition (SICI), cortical silent period (CSP), intracortical facilitation (ICF), long-interval intracortical inhibition (LICl), and cross-activation were measured, using transcranial magnetic stimulation (TMS) targeting the wrist flexor/extensor muscles.

RESULTS: MVIC of the trained arm decreased by $43 \%(P=0.04)$ after training. We observed no changes in MVIC of the untrained hand and in any of the TMS measures (all $P>0.05$ ).

CONCLUSION: A single session of submaximal grip training with or without anodal-high definition-TDCS produces no cross-education of maximal grip force nor does it affect the excitability of the ipsilateral M1.

KEYWORDS: Cross-education | Transcranial direct current stimulation | Ipsilateral motor cortex | Short-interval intracortical inhibition | Strength training 


\section{INTRODUCTION}

Cross-education of muscle strength is the increase in strength of an untrained contralateral limb following a period of unilateral strength training. ${ }^{1}$ The most recent evidence, from a meta-analysis of 31 randomized controlled trials, reported an increase in contralateral strength of $8.2-17.7 \%$ following unilateral strength training of the upper and lower limbs. ${ }^{2}$ As the contralateral increase in strength likely occurs in the absence of any detectable changes in muscle hypertrophy, ${ }^{3}$ the increase in strength of the untrained limb has been attributed to adaptations in the central nervous system. ${ }^{4}$ Several studies have used Transcranial Magnetic Stimulation (TMS) to determine the underlying neurophysiological mechanisms underpinning cross-education following dynamic (concentric and eccentric) $)^{5,6,7}$ and isometric unilateral strength training. ${ }^{8}$ According to TMS studies, the 'untrained' motor cortex (M1), ipsilateral to the trained limb or "ipsilateral M1", plays a critical role in mediating the cross-transfer effect. 5,6,7,9,10 Recent TMS studies found short-term changes in the excitability of the ipsilateral M1 and a reduction in intracortical inhibition following both a single bout and short-term unilateral strength training. 5,6,7,8 Interestingly, similar to the strength training literature, ${ }^{11}$ the ipsilateral TMS responses to cross-education are not consistent. ${ }^{4}$ Several studies have reported no change in the ipsilateral TMS responses, ${ }^{12,13,14}$ whilst others have reported increased ipsilateral M1 excitability and reduced intracortical inhibition. $8,15,16$

Irrespective of the above, it has been suggested that changes in excitability of the ipsilateral M1 could be due to neural mechanisms associated with cross-activation. ${ }^{17,18,19}$ Cross-activation is based upon the observation that unilateral muscle actions result in a bilateral increase in corticospinal excitability 9,17,20,21,22 and a decrease in intracortical inhibition of the ipsilateral M1.18,23 However, a limitation with the previous cross-education TMS studies is that the strength training task and the conditions in which TMS is tested are different. For example, the strength training is usually dynamic, while TMS testing often occurs during isometric actions, thus, there appears to be a lack of specificity when determining the ipsilateral M1 responses following cross-education. ${ }^{24,25}$ Recent experimental data now suggest that studies using TMS to assess the neuromuscular responses to a strength training intervention should use testing modalities that more closely replicate the characteristics of the strength-training intervention. ${ }^{26}$ Interestingly, Hortobágyi et al. ${ }^{8}$ examined the effects of isometric strength training on cross-education and showed increased ipsilateral M1 excitability and inter-hemispheric inhibition when the training and testing conditions were identical. To date, because there is only one crosseducation study where training and testing was the same, a major focus of the present study was to examine the magnitude of cross-activation in a context specific to both the strength training task and the conditions of TMS testing.

To enhance the cross-education effect from strength training, it is possible that the magnitude of cross-activation could be optimized following non-invasive brain stimulation techniques such as transcranial direct current stimulation (TDCS). ${ }^{24,25}$ However, to date, there have only been two studies ${ }^{24,25}$ that have tried to identify the priming effects of ipsilateral anodal- M1 TDCS (ipsilateral to motor training) prior to strength training on the contralateral increases in strength, corticospinal excitability and decrease in inhibition. Interestingly, the findings of these studies suggested that ipsilateral anodal-TDCS could be used as a potential priming technique to enhance the cross-education effect.24,25 
Transcranial direct current stimulation can be used to induce lasting bidirectional excitability changes in the corticospinal tract.27,28,29 Applying a constant low electrical current via small electrodes positioned over the M1, anodal-TDCS is thought to change the threshold of depolarization of neurons and thereby increase corticospinal excitability, contributing to mechanisms of long-term potentiation and long-term depression. ${ }^{27,28,29,30,31}$ Furthermore, TDCS can be used to modulate the excitability of the intrinsic micro-circuits of the M1. A recent systematic review and meta-analysis in healthy subjects indicated that a single session of TDCS decreased short-interval intracortical inhibition (SICI) and increased intracortical facilitation (ICF). ${ }^{32}$ However, despite this encouraging finding, there are no studies that have examined the effects of applying high-definition TDCS prior to or during unilateral strength training. High-definition TDCS provides a more focused electric field when compared to traditional TDCS. ${ }^{33}$ While the previous cross-education TDCS studies have used a conventional form of TDCS with large electrode sizes on the ipsilateral $\mathrm{M} 1,24,25$ a major focus of the current study was to utilize high-definition TDCS to target the M1 using smaller electrodes and increasing the focus of stimulation.

Despite previous research examining the effect of stimulating the ipsilateral M1 during or prior to strength training, ${ }^{24,25}$ there is no study to date that has examined the effects of bilateral M1 (i.e., ipsi- and contralateral M1) stimulation on cross-education. It is now well established by imaging studies that there is a distributed cortical region that is active during ipsilateral limb movement. ${ }^{34,35}$ Prominently, these studies highlighted the role of the ipsilateral M1 in addition to the contralateral M1 for the execution of unilateral motor tasks. For example, Chiou et al. ${ }^{36}$ reported increased bilateral cortical activity of the M1 during unilateral motor tasks, as determined by TMS. This finding suggests a prominent role of the ipsilateral M1 in addition to the contralateral M1 in the execution of unilateral motor tasks.

Neurophysiological support for these possibilities stem from emerging evidence that multi-focal stimulation over functionally-connected regions of the distributed motor network leads to a greater enhancement in corticospinal excitability than single-site stimulation of the M1 alone. ${ }^{37,38}$ For example, Fischer et al. ${ }^{37}$ compared two electrode montages: a traditional montage targeting $\mathrm{M} 1$ and an eight-electrode montage targeting M1 and its associated resting state network. Surprisingly, multifocal TDCS increased corticospinal excitability more than double compared to traditional TDCS. These findings lead to the hypothesis that bilateral TDCS of M1 during strength training could enhance the cross-education of MVIC grip force.

Therefore, the primary aim of this study was to examine the effect of priming the left and right M1 (bilateral) using anodal- high-definition-TDCS concurrently during a single bout of unilateral strength training to determine the efficacy of this technique on modulating the cross-education of strength. A secondary aim was to determine the ipsilateral corticospinal responses, in particular cross-activation following the TDCS conditions. It was

hypothesized that bilateral high-definition-TDCS would increase bilateral cortical excitability and this would facilitate the cross-education of maximal force.

\section{EXPERIMENTAL PROCEDURES}

\section{Study design}

Participants completed the experimental protocol as outlined in Figure 1. After 
obtaining consent, in a randomized cross-over design, participants were exposed to three conditions. Each participant was exposed to 15 minutes of bilateral anodal- high-definitionTDCS, unilateral anodal- high-definition-TDCS and sham-high-definition-TDCS of the M1 during a single strength training (ST) session that involved submaximal isometric grip training (bilateral anodal-high-definition-TDCS + ST, unilateral anodal-high-definition-TDCS + ST and sham high-definition-TDCS + ST, respectively). The order of the conditions was counterbalanced and randomized between participants, with a washout period of 1 week between each condition. ${ }^{24}$. All participants underwent TMS and isometric strength testing (maximum voluntary isometric contraction) of the right and left hand before and after the TDCS and ST interventions (Figure 1). The order of testing hands was randomized.

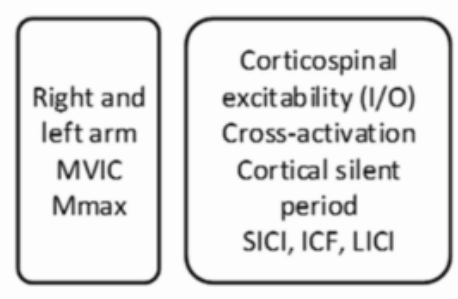

Baseline

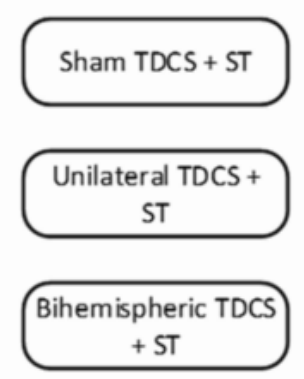

Intervention

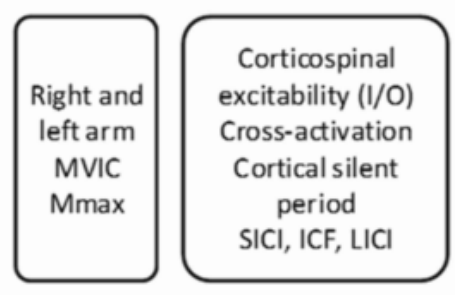

Post-intervention

Figure 1. Experimental protocol. Subjects participated in three experimental sessions. Neurophysiological assessments obtained before and after anodal TDCS and ST. Pre - and post-measures included assessments of peripheral muscle excitability (M $\mathrm{MAx}_{\mathrm{MA}}$ ), maximum voluntary isometric contraction (MVIC), corticospinal excitability input-output curve, cross-activation, corticospinal silent period, short interval intracortical inhibition (SICI), intracortical facilitation (ICF), and ling-interval intracortical inhibition.

\section{Participants}

All volunteers provided written informed consent before participation in the study, which was approved by the Monash University Human Research Ethics Committee (project number: 19936) in accordance with the standards by the Declaration of Helsinki. Sample size calculations were established using previous acute cross-education data in healthy untrained adults. ${ }^{24,25}$ Based upon our previous work, we estimated that ten participants in each condition would provide at least $80 \%$ power (95\% confidence interval) to detect an $8.5 \%$ cross-transfer of strength and $8 \%$ increase in ipsilateral corticospinal excitability using a repeated measures design ( $G^{*}$ Power 3.1 .7 software). In this regard, 12 participants (8 women and 4 men), aged 18-50 years (mean $\pm S D=34.34 \pm 8.46$ years) and body mass index of $23.72 \pm 4.10 \mathrm{~kg} \cdot \mathrm{m}^{-2}$ volunteered to participate in the study. All volunteers provided written informed consent before participation in the study. All participants were right-hand dominant as determined by the Edinburgh Handedness Inventory ${ }^{39}$ with a Laterality Quotient score of $92 \pm 4$, and had not participated in strength training for a minimum of 12 months. Exclusion criteria included a history of any psychiatric or neurological disorder, seizure, any musculoskeletal pain or disorder especially in upper quadrant, or current pregnancy. Before the experiments, all participants completed the adult safety screening questionnaire to determine their suitability for TMS and TDCS. 40 


\section{Isometric strength testing}

Maximum voluntary isometric contraction (MVIC) force was measured using a grip force transducer (ADInstruments MLT004/ST, Australia). Participants were instructed to sit on a chair with an armrest, the shoulder abducted at $45^{\circ}$, elbow flexed at $90^{\circ}$ and the forearms rested on a chair rest whilst their hand was in a neutral position. The participant was then instructed to squeeze the transducer by cylindrical power grip as forcefully as possible for three seconds. Three attempts, with a 2-minute rest between each attempt, were performed. The standard criteria for measurement of MVICs were fulfilled and included a period of familiarization (before data collection); verbal encouragement was provided by the investigators during MVICs, and real-time force feedback was provided to the participants on a computer screen directly in front of them.

\section{Strength Training Protocol}

Participants completed supervised submaximal grip training while receiving anodal-TDCS and sham-TDCS. Using the same setup as the MVIC testing, participants completed a submaximal cylindrical power gripping task of the right hand. Participants were instructed to sit on a chair with an armrest, their shoulder abducted $45^{\circ}$, elbow flexed at $90^{\circ}$ and their forearms rested on a chair rest whilst their hand was in a neutral position. The participant was then instructed to squeeze the transducer at $70 \%$ of MVIC grip force (force feedback was provided to each participant by setting force targets [a running line] that represented each participant's $70 \%$ MVIC) and to perform four sets of 10 isometric contractions held steadily for 5 seconds. ${ }^{41}$ The recovery interval between contractions and sets were 10 and 90 seconds, respectively. The participants received visual force feedback for the duration of the strength training session through a monitor in front of them. The participants were instructed to rapidly produce force at contraction onset and match their force output as closely as possible to the force tracing during each 5 second contraction. During each contraction, the participants were required to relax the contralateral limb, whereby mirror surface electromyography (sEMG) was recorded. sEMG activity was recorded from the left untrained wrist flexors and extensors to determine the magnitude of mirror sEMG activity as a percent of maximum root-mean-square (rms) EMG. Maximum rms EMG was obtained during maximum grip testing of the left untrained limb during baseline testing conditions.

\section{High definition -Transcranial Direct Current Stimulation}

For anodal-high-definition-TDCS, $3.14 \mathrm{~cm}^{2}$ PiStim electrodes (Neuroelectrics, Barcelona, Spain) were embedded within an electroencephalography ${ }^{42}$ cap using the 10 20 electrode placement system. The configuration was chosen based on previous published methodologies. ${ }^{43,44}$ In the unilateral M1 condition, ipsilateral to the training side, the anode was placed over C4 and the return electrodes (cathodes) at FC6, T8 and CP6. In the bilateral $\mathrm{M} 1$ condition, anodes were placed over $\mathrm{C} 3$ and $\mathrm{C} 4$, with return electrodes at FC5, T7, CP5, FC6, T8 and CP6. Anodal-TDCS (electrodes placed at C3 and C4) was delivered at $1.5 \mathrm{~mA}$ (current density of $0.478 \mathrm{~mA} / \mathrm{cm}^{2}$ ) for $15 \mathrm{~min}$ (30 second ramp-up and 10 second ramp-down period at the beginning and end of stimulation, respectively). In the sham condition, participants were pseudorandomized to receive either unilateral M1 or bilateral M1 stimulation. In the sham condition, the current was ramped up for 30 seconds 
and then ramped down to $0 \mathrm{~mA}$ and then, in the last 10 seconds, the stimulus returned and ramped down to $0 \mathrm{~mA}$ to give the illusion of stimulation to the participant.

\section{Surface electromyography}

The area of electrode placement was shaved to remove fine hair, rubbed with an abrasive skin gel to remove dead skin, and then cleaned with $70 \%$ isopropyl alcohol. sEMG was recorded from the right flexor carpi radialis (FCR) and extensor carpi radialis brevis (ECRB) muscles using bipolar Ag-AgCl electrodes according to SENIAM guidelines. The sites of measurement for the FCR and ECRB were determined by marking the skin two-thirds of the distance between the medial epicondyle of humerus and base of metacarpals II and III, and lateral epicondyle of humerus and dorsal surface of the base of metacarpals II and III, respectively. This mark was then extended to the point of the muscle bulk, and the electrodes were placed $2 \mathrm{~cm}$ apart, with a ground electrode secured on the dorsum of the wrist. sEMG signals were measured with an impedance meter to ensure impedance did not exceed $10 \mathrm{k} \Omega$ prior to testing. sEMG signals were amplified $(\times 1,000)$, bandpass filtered (high pass at $13 \mathrm{hz}$, low pass at 1,000 Hz), digitized online at $2 \mathrm{kHz}$ for 1 second, recorded and analysed using Powerlab 4/35 (ADInstruments, Australia).

\section{Transcranial magnetic stimulation}

Transcranial magnetic stimulation was delivered to the untrained limb using a Magstim $200^{2}$ stimulator (Magstim Co, United Kingdom) and a single figure-of-eight coil (external diameter of each loop $70 \mathrm{~mm}$ ). Sites near the estimated centre of the right FCR and ECRB area (motor hotspot) were explored to determine the cortical region that produced the largest MEP amplitude. Once the hotspots were identified, active motor threshold (AMT) was established as the intensity at which at least 5 of 10 stimuli produced MEP amplitudes of greater than $200 \mu \mathrm{V}^{45}$ during a low-level isometric contraction. After the TDCS and unilateral strength-training intervention, AMT was retested and adjusted if required. To ensure all stimuli were delivered to the optimal motor hotspot throughout testing, the position of the coil was marked with semi-permanent ink on the motor hotspots.

All stimuli were delivered during low-level isometric contraction of the FCR and ECRB muscles, which were performed by maintaining $20^{\circ}$ of wrist flexion and extension..$^{15}$ These positions equated to $1 \pm 0.2 \%$ of rmsEMG, and consistent muscle activation was confirmed by recording pre-stimulus rmsEMG throughout testing..$^{15}$ In order to ensure the optimal joint position was obtained by the participants, joint angle was measured with a twin axis electronic goniometer (ADInstruments, Australia), with visual feedback provided on a screen visible to both the participant and the researcher. ${ }^{46}$

We examined both "single-pulse TMS" which is used to assess corticospinal excitability and "paired pulse TMS", to assess $\mathrm{GABA}_{A}$-mediated $\mathrm{SICl}, \mathrm{GABA}_{B}$-mediated $\mathrm{LICl}$ and glutamatergic ICF.

\section{Single-pulse TMS induced MEPs}

Single-pulse TMS was used to assess corticospinal excitability of the untrained FCR and ECRB muscles. Stimulus-response curves were constructed to determine corticospinal excitability (MEP amplitude) and corticospinal inhibition (cortical silent period duration) for the untrained contralateral FCR. The stimulus intensities used to establish the TMS recruitment curves were determined for each individual according to their AMT before 
and after the training intervention. At each stimulus intensity, 10 stimuli were delivered over the right $\mathrm{M} 1$, with the percentage of stimulator output applied in a pseudo-randomized manner. Specifically, 10 stimuli were given at stimulus intensities of $120,130,150$, and $170 \%$ of AMT.47 Each stimulus was delivered in random intervals every 10 to 12 seconds to avoid stimulus anticipation, and 30 seconds rest was provided between each set of stimuli to reduce the possibility of muscle fatigue. With the same protocol as the agonist muscle of the untrained limb, but with a different hot spot, we also assessed corticospinal excitability in the antagonist muscle (ECRB) of the untrained limb using single-pulse TMS induced MEPs at $120 \%$ and $130 \%$ of AMT.

\section{Intracortical excitability and inhibition}

Intracortical excitability and inhibition were measured in the left FCR and ECRB by a TMS paired-pulse protocol, including 30 stimuli, and interstimulus intervals (ISIs) of 3, 10, and $100 \mathrm{~ms}$ in random order. In this protocol, short-intracortical inhibition (SICl, $3 \mathrm{~ms})$, and intracortical facilitation (ICF, $10 \mathrm{~ms}$ ) were assessed by combining a subthreshold conditioning stimulus (CS: 80\% AMT) with a suprathreshold test stimulus (TS: $120 \%$ AMT). Long-intracortical inhibition ( $\mathrm{LICl}, 100 \mathrm{~ms}$ ) was assessed by using two suprathreshold test stimuli (TS: $120 \%$ AMT).

\section{Cross-activation}

To determine cross-activation of the FCR and ECRB, three stimuli were delivered to the right M1 hotspot at $130 \%$ AMT during a gripping MVIC task. Participants were required to meet their target MVIC, with maximal force feedback provided. On average, participants completed three MVICs with 60 seconds rest in between each. The condition in which cross-activation was obtained was identical to the condition of the strengthtraining intervention and the condition in which MVIC force was determined. 8

\section{Maximum Compound Muscle Action Potential}

Direct muscle responses were obtained from the left untrained FCR and ECRB muscles by supramaximal electrical stimulation (pulse width $200 \mu \mathrm{s}$ ) of the brachial plexus, while holding $1 \% \pm 0.2 \%$ of the FCR and ECRB rmsEMG (DS7A, Digitimer, UK). This low level of muscle activity was used to match the conditions under which TMS was delivered. ${ }^{25}$ The stimulation site that produced the largest M-wave was located by positioning the bipolar electrodes on Erb's point. An increase in current strength was applied to the brachial plexus until there was no further increase observed in the amplitude of the sEMG response (MMAX). To ensure maximal responses, the current was increased an additional $20 \%$, and the average $\mathrm{M}_{\text {MAX }}$ was obtained from five stimuli, with a period of 6-9 seconds separating each stimulus. MMAX was recorded at baseline and following the TDCS and strength training intervention to control for possible changes in peripheral muscle excitability that could influence MEP amplitude.

\section{Data Analysis}

Pre-stimulus rmsEMG activity was determined in the untrained FCR and ECRB muscles $100 \mathrm{~ms}$ prior to each TMS stimulus during pre- and post-testing. Any trial in which pre-stimulus rmsEMG exceeded $5 \%$ of maximal rmsEMG was discarded and the trial was repeated. The peak-to-peak amplitude of MEPs evoked as a result of stimulation was 
measured in the left FCR and ECRB muscles contralateral to the cortex being stimulated in the period 10-50 ms after stimulation. MEP amplitudes were analysed (LabChart 8 software, ADInstruments, Australia) after each stimulus was automatically flagged with a cursor, providing peak-to-peak values in $\mu \mathrm{V}$, averaged and normalized to the maximum compound wave (MMAX), and multiplied by 100.

Silent period durations were obtained from single-pulse stimuli delivered at 120$170 \%$ AMT during a low-level isometric contraction (1.0 $\pm 0.2 \%$ of maximal rmsEMG of the untrained FCR and ECRB muscles). The duration between the onset of the MEP and the resolution of background SEMG was visually inspected and manually cursored, with the experimenter blinded to each condition and the average from ten stimuli used to determine silent period duration. 48

In addition, the total area under the recruitment curve (AURC) was calculated with the method of trapezoidal integration using the data collected during the construction of corticospinal excitability and inhibition recruitment curves for the untrained left FCR muscle. The data obtained from the AURC are presented as arbitrary units. ${ }^{20}$

\section{Statistical Analysis}

The target sample size was based on a priori calculation, which included the observed effect size from our previous experiments. ${ }^{24,25}$ In previous experiments, samples sizes around 10 have been adequate to observe statistically significant changes in motor-evoked potentials (MEPs) and $\mathrm{SICI}$ following unilateral strength training. ${ }^{49}$

All data were first screened to ensure they were normally distributed. To have sufficient data to test for questions of normality, all data from baseline MEPs, SICI, ICF, $\mathrm{LICl}, \mathrm{MVIC}$ trials were used to establish the distributional properties. There were no variable z-scores of skewness or kurtosis. A one-way analysis of variance (ANOVA) was used to compare baseline neurophysiological measures between the three intervention conditions (sham high-definition-TDCS + ST, unilateral high-definition-TDCS + ST, and bilateral high-definition-TDCS + ST). A split-plot in time repeated measures ANOVA was used to calculate the effect of each CONDITION (sham high-definition-TDCS + ST, unilateral anodal high-definition-TDCS + ST and bilateral anodal high-definition-TDCS + ST) on voluntary strength and the indices of cortical plasticity (AURC for corticospinal excitability, AURC for corticospinal inhibition, ICF, SICI, LICl and cross-activation). Significant main effects and interactions were examined further using one-way repeated measure ANOVAs and Bonferoni-adjusted pairwise comparisons. To assess the magnitude of effects, partial eta-squared $\left(\eta_{p}^{2}\right)$ were calculated. There were no outliers, as assessed by examination of studentized residuals for values greater than \pm 3 . All data are presented as mean $\pm \mathrm{SD}$.

\section{RESULTS}

\section{Pre-stimulus rmsEMG and $M_{\text {MAX }}$}

Table 1 presents the mean ( \pm SD) AMT stimulus intensity, MMAX amplitude, singlepulse and paired-pulse pre-stimulus rmsEMG before and after sham high-definition-TDCS + ST, unilateral anodal high-definition-TDCS + ST, and bilateral anodal high-definitionTDCS + ST for FCR and ECRB muscles. Single-pulse and paired-pulse pre-stimulus rmsEMG, AMT stimulation intensity, and $\mathrm{M}_{\mathrm{MAX}}$ were similar between the three conditions at 
baseline for both FCR and ECRB muscles $(P>0.05)$, indicating consistent values across the three conditions prior to the intervention. There were no TIME or TIME $\times$ CONDITION interactions detected for single and paired-pulse pre-stimulus rmsEMG, AMT stimulation intensity, or $\mathrm{M}_{\operatorname{MAX}}$ for both the FCR and ECRB muscles $(P>0.05)$.

\section{Maximal Voluntary Isometric Contraction Force}

Table 2 presents the MVIC force for the right and left FCR and ECRB muscles before and after sham-high-definition-TDCS + ST, unilateral anodal-high-definition-TDCS + ST, and bilateral anodal-high-definition-TDCS + ST. There were no differences in MVIC force at baseline between sham-high-definition-TDCS + ST, unilateral anodal-highdefinition-TDCS + ST, and bilateral anodal-high-definition-TDCS + ST of the left $\left(\mathrm{F}_{(2,33)}=\right.$ $0.003 ; P=0.99)$ and right $\left(F_{(2,33)}=0.03 ; P=0.96\right)$ limbs. Repeated measure two-way ANOVA revealed that there was a significant main effect for $\operatorname{TIME}\left(\mathrm{F}_{(1,11)}=8.14, P=0.02\right.$, $\left.\eta_{\mathrm{p}}{ }^{2}=0.43\right)$ and a TIME $\times$ CONDITION interaction $\left(\mathrm{F}_{(2,22)}=5.47 ; P=0.01, \eta_{\mathrm{p}}{ }^{2}=0.33\right)$. Repeated measure one-way ANOVA revealed that there was a significant difference between the trained right limb MVIC force post unilateral anodal-high-definition-TDCS + ST and sham-high-definition-TDCS + ST $(P=0.008)$, with a mean difference between conditions of $-41.5 \mathrm{~N}(95 \% \mathrm{Cl},-71.6$ to -11.3$)$. For the untrained left limb, repeated measure two-way ANOVA revealed that there were no significant main effects for TIME $\left(F_{(1,11)}=0.10, P=0.76, \eta_{p}^{2}=0.01\right)$ or any TIME $\times$ CONDITION interactions $\left(F_{(2,22)}=0.18\right.$, $P=0.83, \eta_{\mathrm{p}}^{2}=0.02$ ).

Table 1 - Mean $( \pm$ SD) for AMT stimulus intensity, MmAx and single-pulse TMS pre-stimulus rmsEMG before and after sham TDCS + ST, unilateral TDCS + ST, and bilateral TDCS + ST

\begin{tabular}{|c|c|c|c|c|c|c|}
\hline & $\begin{array}{c}\text { Sham TDCS } \\
+ \text { ST PRE }\end{array}$ & $\begin{array}{c}\text { Sham TDCS } \\
+ \text { ST post }\end{array}$ & $\begin{array}{c}\text { Unilateral TDCS } \\
+ \text { ST PRE }\end{array}$ & $\begin{array}{c}\text { Unilateral TDCS } \\
+ \text { +ST POST }\end{array}$ & $\begin{array}{c}\text { Bilateral TDCS } \\
+ \text { ST PRE }\end{array}$ & $\begin{array}{c}\text { Bilateral TDCS } \\
+ \text { ST post }\end{array}$ \\
\hline FCR AMT SI (\%) & $41 \pm 6$ & $42 \pm 6$ & $41 \pm 5$ & $41 \pm 4$ & $40 \pm 6$ & $40 \pm 7$ \\
\hline ECR AMT SI (\%) & $40 \pm 6$ & $39 \pm 5$ & $40 \pm 6$ & $40 \pm 5$ & $39 \pm 5$ & $39 \pm 4$ \\
\hline $\begin{array}{l}\text { FCR SP rmsEMG (\% } \\
\text { rmsEMG }\end{array}$ & $1.2 \pm 1$ & $0.94 \pm 0.46$ & $1 \pm 0.62$ & $1 \pm 0.70$ & $0.95 \pm 0.41$ & $1.20 \pm 0.74$ \\
\hline $\begin{array}{l}\text { FCR PP rmsEMG (\% } \\
\text { rmsEMG }\end{array}$ & $1.3 \pm 1.3$ & $0.95 \pm 0.60$ & $1.14 \pm 0.86$ & $1 \pm 0.75$ & $0.98 \pm 0.57$ & $1 \pm 0.76$ \\
\hline $\begin{array}{l}\text { ECR SP rmsEMG (\% } \\
\text { rmsEMG }\end{array}$ & $1.5 \pm 0.75$ & $2.3 \pm 0.75$ & $1.3 \pm 1$ & $1.2 \pm 0.50$ & $1.6 \pm 1.2$ & $1.5 \pm 0.92$ \\
\hline $\begin{array}{c}\text { ECR PP rmsEMG (\% } \\
\text { rmsEMG }\end{array}$ & $1.2 \pm 0.65$ & $1 \pm 0.68$ & $1.1 \pm 0.87$ & $0.93 \pm 0.44$ & $1.2 \pm 0.80$ & $1.1 \pm 0.77$ \\
\hline FCR $M_{\operatorname{MAX}}(\mathrm{mV})$ & $4.1 \pm 1.2$ & $5.1 \pm 2$ & $5 \pm 1.7$ & $5.1 \pm 1.9$ & $4.4 \pm 1.09$ & $4.3 \pm 1.2$ \\
\hline$E C R M_{\max }(m V)$ & $4.6 \pm 2.5$ & $5.1 \pm 3$ & $5.3 \pm 2.9$ & $5.9 \pm 3.5$ & $5.8 \pm 3.6$ & $5.9 \pm 4.2$ \\
\hline
\end{tabular}

TDCS = transcranial direct current stimulation, AMT SI = active motor threshold stimulus intensity, TMS = transcranial magnetic stimulation, FCR = flexor carpi radialis, FCR SP rmsEMG = flexor carpi radialis single-pulse root mean square electromyography, FCR PP rmsEMG = flexor carpi radialis paired-pulse root mean square electromyography, ECR = extensor carpi radialis brevis, ECR SP rmsEMG = extensor carpi radialis brevis single-pulse root mean square electromyography, ECR PP rmsEMG = extensor carpi radialis brevis paired-pulse root mean square electromyography, rmsEMGMAX = root mean squared electromyography maximum. 
Table 2 - Mean $( \pm$ SD) for right and left MVIC before and after sham TDCS + ST, unilateral TDCS + ST, and bilateral TDCS + ST

\begin{tabular}{|c|c|c|c|c|c|c|}
\hline & $\begin{array}{c}\text { Sham TDCS } \\
+ \text { STPRE }\end{array}$ & $\begin{array}{c}\text { Sham TDCS } \\
\text { + ST Post }\end{array}$ & $\begin{array}{c}\text { Unilateral TDCS } \\
+ \text { STPRE }\end{array}$ & $\begin{array}{c}\text { Unilateral TDCS } \\
\text { + STpost }\end{array}$ & $\begin{array}{c}\text { Bilateral TDCS } \\
+ \text { STPRE }\end{array}$ & $\begin{array}{c}\text { Bilateral TDCS } \\
\text { + STPOST }\end{array}$ \\
\hline Right MVIC (N) & $382 \pm 156$ & $326 \pm 129$ & $383 \pm 121$ & $368 \pm 120^{*}$ & $395 \pm 130$ & $352 \pm 127$ \\
\hline Left MVIC (N) & $355 \pm 145$ & $359 \pm 153$ & $358 \pm 147$ & $362 \pm 153$ & $360 \pm 153$ & $357 \pm 153$ \\
\hline
\end{tabular}

MEP AURC FCR

The MEP AURC for the untrained FCR muscle was similar between conditions at baseline $\left(F_{(2,20)}=1.43, P=0.26\right)$. There were no main effects for $\operatorname{TIME}\left(F_{(1,8)}=0.05, P=\right.$ $\left.0.83, \eta_{p}^{2}=0.01\right)$ or any TIME $\times$ CONDITION interactions detected $\left(F_{(2,16)}=1.14 ; P=0.33\right.$, $\left.\eta_{\mathrm{p}}{ }^{2}=0.13\right)($ Table 3 , and Supplementary material Figure 2).

Table 3 - Mean $( \pm$ SD) for MEP AURC and Silent Period AURC, ICF, SICI and LICI before and after before and after sham TDCS + ST, unilateral TDCS $+\mathrm{ST}$, and bilateral TDCS + ST

\begin{tabular}{|c|c|c|c|c|c|c|}
\hline & $\begin{array}{c}\text { Sham TDCS } \\
+ \text { STPRE }\end{array}$ & $\begin{array}{c}\text { Sham TDCS } \\
+ \text { STPost }\end{array}$ & $\begin{array}{c}\text { Unilateral TDCS } \\
+ \text { STPRE }\end{array}$ & $\begin{array}{c}\text { Unilateral TDCS } \\
+ \text { STPOST }\end{array}$ & $\begin{array}{c}\text { Bilateral TDCS } \\
+ \text { ST PRE }\end{array}$ & $\begin{array}{c}\text { Bilateral TDCS } \\
\text { + STPost }\end{array}$ \\
\hline $\begin{array}{c}\text { MEP amplitude } \\
\text { AURC (Au) } \\
\text { Silent Period }\end{array}$ & $1532 \pm 576$ & $1284 \pm 650$ & $1226 \pm 567$ & $1396 \pm 1009$ & $1452 \pm 706$ & $1333 \pm 708$ \\
\hline $\begin{array}{c}\text { Duration AURC } \\
\text { (Au) }\end{array}$ & $4.7 \pm 1.1$ & $4.8 \pm 0.9$ & $4.5 \pm 1.3$ & $4.7 \pm 1.3$ & $4.3 \pm 0.8$ & $4.2 \pm 0.8$ \\
\hline $\begin{array}{l}\text { ICF (\% Test } \\
\text { Response) }\end{array}$ & $150.8 \pm 61.9$ & $148.3 \pm 63.5$ & $116.2 \pm 10.9$ & $142.1 \pm 70.8$ & $125.8 \pm 35.0$ & $122.8 \pm 21.8$ \\
\hline $\begin{array}{l}\text { SICI (\% Test } \\
\text { Response) }\end{array}$ & $52.5 \pm 23.2$ & $41.9 \pm 22.2$ & $43.2 \pm 19.4$ & $43.9 \pm 22.8$ & $52.2 \pm 23.4$ & $56.1 \pm 20.5$ \\
\hline $\begin{array}{l}\text { LICI (\% Test } \\
\text { Response) }\end{array}$ & $40.77 \pm 18.3$ & $52.0 \pm 19.1$ & $48.4 \pm 21.1$ & $48.9 \pm 25.1$ & $42.4 \pm 27.5$ & $40.0 \pm 22.5$ \\
\hline
\end{tabular}

$\mathrm{MEP}=$ motor-evoked potentials, $\mathrm{AURC}=$ area under the recruitment curve, $\mathrm{ICF}=$ intracortical facilitation, $\mathrm{SICl}=$ short-interval cortical inhibition, $\mathrm{LICl}=$ long-interval cortical inhibition.

\section{Cortical Silent Period Duration AURC}

The cortical silent period AURC for the FCR muscle was similar between conditions at baseline $\left(\mathrm{F}_{(2,20)}=0.15 ; P=0.87\right)$. There were no main effects for $\operatorname{TIME}\left(\mathrm{F}_{(1,8)}\right.$ $\left.=0.43, P=0.53, \eta_{\mathrm{p}}^{2}=0.05\right)$ or TIME $\times$ CONDITION interactions detected $\left(\mathrm{F}_{(2,16)}=1.96 ; P\right.$ $=0.17, \eta_{p}^{2}=0.2 ;$ Supplementary material Figure 3 ).

\section{Intracortical Facilitation}

ICF for the FCR muscle was inhibited in six participants and thus these participants were excluded from the analysis. ICF was similar between conditions at baseline $\left(F_{(2,10)}=1.719, P=0.23\right)$; however, there were no main effects for $\operatorname{TIME}\left(F_{(1,5)}=\right.$ $\left.8.01, P=0.07, \eta_{p}^{2}=0.72\right)$ or any TIME $\times$ CONDITION interactions detected $\left(F_{(2,10)}=1.67\right.$; $P=0.27, \eta_{\mathrm{p}}{ }^{2}=0.35$; Supplementary material Figure 4).

\section{Short-Interval Cortical Inhibition}

The $\mathrm{SICI}$ ratio was facilitated in three participants, thus they were excluded from further analysis. SICI was similar between conditions at baseline $\left(\mathrm{F}_{(2,16)}=2.84, P=0.09\right)$ and there were no main effects for TIME $\left(F_{(1,8)}=0.99, P=0.36, \eta_{p}^{2}=0.14\right)$ or any TIME $\times$ 
CONDITION interactions detected $\left(F_{(2,16)}=1.14, P=0.35, \eta_{p}^{2}=0.16\right.$; Supplementary material Figure 5).

\section{Long-Interval Cortical Inhibition}

The data of three participants were facilitated and were excluded from further analysis. The $\mathrm{LICl}$ ratio for the $\mathrm{FCR}$ muscle was similar between conditions at baseline $\left(\mathrm{F}_{(2}\right.$, 16) $=1.32 ; P=0.30)$ and there were no main effects for time $\left(F_{(1,8)}=3.34, P=0.11, \eta_{p}{ }^{2}=\right.$ $0.30)$ or any TIME $\times$ CONDITION interactions detected $\left(F_{(2,16)}=0.29, P=0.75, \eta_{p}{ }^{2}=0.04\right.$; Supplementary material Figure 6).

\section{Cross activation}

Cross-activation for the FCR muscle was similar between conditions at baseline $\left(F_{(2,20)}=0.01, P=0.96\right)$ and there were no main effects for time $\left(F_{(1,10)}=2.93, P=0.12, \eta_{p}{ }^{2}\right.$ $=0.23)$ or any TIME $\times$ CONDITION interactions detected $\left(F_{(2,20)}=1.24, P=0.31, \eta_{p}{ }^{2}=\right.$ 0.11 ; Supplementary material Figure 7).

\section{Mirror sEMG}

We also examined whether submaximal grip training of the right hand affected the degree of mirror sEMG activity in the contralateral untrained left wrist flexors and extensors. Averaged across the three intervention conditions, there were no differences in mirror sEMG activity for the flexor muscles $\left(2.07 \%, 95 \% \mathrm{Cl}, 0.98\right.$ to $3.15 \%, \mathrm{~F}_{(2,22)}=0.23, P=$ $\left.0.80, \eta_{p}{ }^{2}=0.02\right)$ or extensors muscles $\left(1.89 \%, 95 \% \mathrm{Cl}, 0.93\right.$ to $2.86 \%, \mathrm{~F}_{(2,22)}=0.56, P=$ $\left.0.58, \eta_{p}^{2}=0.05\right)$.

\section{ECR muscle}

\section{Corticospinal excitability}

Table 4 presents the MEP, ICF and SICI data for left ECRB muscles before and after sham-high-definition-TDCS + ST, unilateral anodal-high-definition-TDCS + ST, and bilateral anodal-high-definition-TDCS + ST. The MEP amplitude at 130\% AMT for the ECRB muscle was similar between conditions at baseline $\left(F_{(2,22)}=1.40, P=0.27\right)$. There was no main effect for time $\left(F_{(1,11)}=0.02, P=0.89, \eta_{p}{ }^{2}=0.00\right)$ or any TIME $\times$ CONDITION interaction detected $\left(F_{(2,22)}=1.31, P=0.29, \eta_{p}^{2}=0.11\right.$; Table 4).

\section{Short-Interval Intracortical Inhibition}

The SICI ratio was facilitated in nine participants, thus they were excluded from further analysis. Due to small numbers of included data, no further analysis was performed.

Long-Interval Intracortical Inhibition

The LICI ratio was facilitated in all of the participants, thus this data was excluded from further analysis.

\section{Intracortical Facilitation}

The ICF ratio was inhibited in nine participants, thus they were excluded from further analysis. Due to small numbers of included data, no further analysis was performed. 
Table 4 - Mean $( \pm S D)$ for MEP, ICF, SICl of the ECR before and after before and after sham TDCS + ST, unilateral TDCS + ST, and bilateral TDCS + ST

\begin{tabular}{ccccccc}
\hline & $\begin{array}{c}\text { Sham TDCS } \\
\text { + STPRE }\end{array}$ & $\begin{array}{c}\text { Sham TDCS } \\
\text { + STPOST }\end{array}$ & $\begin{array}{c}\text { Unilateral TDCS } \\
\text { + STPRE }\end{array}$ & $\begin{array}{c}\text { Unilateral TDCS } \\
\text { + STPOST }\end{array}$ & $\begin{array}{c}\text { Bilateral TDCS } \\
\text { + STPRE }\end{array}$ & $\begin{array}{c}\text { Bilateral TDCS } \\
\text { + STPost }\end{array}$ \\
\hline $\begin{array}{c}\text { MEP (\% of MmAx) } \\
\text { ICF (\% Test }\end{array}$ & $23.6 \pm 9.5$ & $24.2 \pm 15.6$ & $16.5 \pm 10$ & $17.9 \pm 7.6$ & $18.2 \pm 12.7$ & $14.52 \pm 5.7$ \\
$\begin{array}{c}\text { Response) } \\
\text { SICl (\% Test } \\
\text { Response) }\end{array}$ & $163 \pm 72.6$ & $107.9 \pm 10.3$ & $144.4 \pm 34.9$ & $132.5 \pm 22.9$ & $162.3 \pm 65.7$ & $127.1 \pm 24.18$ \\
& $56.5 \pm 27.3$ & $54.2 \pm 20.6$ & $65.1 \pm 16.4$ & $71.7 \pm 22.5$ & $68.47 \pm 23.5$ & $69.5 \pm 20.71$ \\
\hline
\end{tabular}

$\mathrm{MEP}=$ motor-evoked potentials, $\mathrm{ICF}=$ intracortical facilitation, $\mathrm{SICl}=$ short-interval cortical inhibition .

\section{Cross activation}

Cross-activation for the ECRB muscle was similar between conditions at baseline $\left(\mathrm{F}_{(2,22)}=0.16, P=0.85\right)$. There was no main effect for $\operatorname{TIME}\left(\mathrm{F}_{(1,11)}=2.33, P=0.17, \eta_{\mathrm{p}}{ }^{2}=\right.$ $0.23)$ or any TIME $\times$ CONDITION interaction detected $\left(F_{(2,22)}=0.35, P=0.71, \eta_{p}^{2}=0.04\right)$.

\section{DISCUSSION}

The primary aim of this study was to determine if one session of submaximal grip strength training produces a cross-education effect and whether this cross-education effect could be enhanced by anodal-high-definition-TDCS. The secondary aim was to determine if measures of ipsilateral M1 excitability could account for any cross-education effects, enhanced or not by anodal-high-definition-TDCS. Overall, we found no differences in muscle strength and/or changes in corticospinal excitability and inhibition across any of the high-definition-TDCS conditions for the untrained limb. Our results indicate that a single session of isometric grip training and anodal-high-definition-TDCS had no effect on modulating the excitability of the intrinsic micro-circuitry of the M1, which is in contrast to previous anodal-TDCS strength training studies. ${ }^{24,25,50}$ We suggest that there are several factors that have contributed to the discrepancies between the results of the current study and of the previous studies listed above.

\section{Acute submaximal isometric grip training does not provoke cross-education}

Practicing ballistic motor skills with one limb can improve ballistic motor performance of the contralateral untrained limb following a single training session. ${ }^{51,52,53}$ Further, unlike the ballistic motor skill training literature, the present findings suggest that submaximal grip training of one limb is insufficient to induce cross-education. This finding is in agreement with previous cross-education studies whereby a single session of strength training of the FDI and biceps brachii was inadequate to provoke cross-education. 8,41 Similar to the strength training data, Mason et al. ${ }^{54}$ observed significant increases in maximal strength following three strength training sessions, whilst Hortobágyi et al. ${ }^{8}$ showed that at least five training sessions were required to induce cross-education, which is a similar finding to Carr et al. ${ }^{41}$, who reported increased contralateral strength following six training sessions. However, it was hypothesized that the application of anodal-highdefinition-TDCS may enhance the cross-education effect which has previously been reported following a single session. ${ }^{24,25}$

The lack of cross-education could be due to the type of strength training employed (submaximal isometric grip training) and the fact that the strength of the trained limb decreased following training. This is important for two reasons. First, in agreement with 
determining the time-course that provokes cross-education, the magnitude of strength gained in the untrained limb is related to the amount of strength gained in the trained limb. ${ }^{2}$ Thus, the decrease in MVIC grip force of the trained limb, most likely negated any crosseducation. Secondly, we reported no changes in cross-activation following any of the conditions, but particularly following the sham condition. There is experimental data to suggest that, as a result of fatigue, there is an increase in bilateral cortical activity. ${ }^{55}$ Despite the fact that MVIC grip force was reduced following all conditions and that we observed no change in cross-activation, there may be other mechanisms at play that modulate cross-education. For example, it is possible that ipsilateral MEPs induced by cross-activation remained unchanged due to a modification in the balance between maximal force production (during cross-activation) and recruitment gradation of motor units of the FCR muscle. In distal upper limb muscles, such as the FCR, only a limited number of additional motor units become activated at force levels at or higher than $50 \%$ MVIC. ${ }^{56}$ Thus, at higher force levels, and given the time-course of after-hyperpolarization (i.e., an exponential decline until the resting membrane potential), the chance that a TMS pulse reaches a motoneuron during the refractory period or at the initial segment of the afterhyperpolarization increases, suggesting that the motoneuron pool is less likely to be responsive to the TMS pulse.

Nevertheless, the type of exercise employed may also explain the null finding of this study. $8,57,58$ Several studies have examined the corticospinal responses in the ipsilateral M1 following a single session of strength training. $8,57,58,59,60$ Collectively, the results from these studies are inconsistent, with some studies reporting an increase in corticospinal excitability and a decrease in $\mathrm{SICl} 57,58$ following dynamic training (biceps curls), whilst others have reported no changes in CSE, SICI, CSP, ICF and interhemispheric inhibition of ipsilateral M1 following isometric strength training. ${ }^{8,59,61}$ The result of our study is similar to the latter studies, suggesting that a single session of submaximal grip training had no effect on the ipsilateral corticospinal responses. Previously, it has been suggested that dynamic rather than isometric contractions activate the ipsilateral M1 more strongly and sustainably throughout the contraction. ${ }^{10}$ Interestingly, two previous studies that used anodal-TDCS with strength training also used a dynamic, rather than an isometric training protocol. ${ }^{24,25}$ Thus, the absence of a cross-education effect could be related to the training task employed and likely explains the discrepancy between the current result and the previous ones. ${ }^{24,25}$ It has previously been reported that crossactivation is greater following dynamic compared to isometric muscle actions, with the suggestion that there could be greater neural resources required for programming and planning of dynamic compared to isometric training. ${ }^{62}$ It could also be related to inhibitory and facilitatory influences from the dorsal premotor and posterior parietal cortices in the contralateral M1 and ipsilateral M1.63

\section{High-definition multi-focal TDCS does not affect measures of ipsilateral M1 excitability}

We hypothesized that if high-definition-TDCS provoked cross-education, then changes in ipsilateral M1 excitability may account for this effect. However, our findings are in contrast with the previously reported findings whereby TDCS facilitated measures of ipsilateral M1 excitability and provoked cross-education. ${ }^{24,25}$ 
There are several reasons that may explain these null effects. First, there is experimental data available that suggests a lack of change in the ipsilateral M1 responses could be due to response variability following TDCS. $.44,65,66$ While anodal-TDCS has been proposed as a method to induce corticospinal excitability, there is a body of evidence that clearly shows, even when the same TDCS protocols are used, that there is a significant individual variability in the corticospinal responses, with about one-half of the subjects failing to respond to the stimulation in the expected manner. ${ }^{64,65,66}$ Indeed, our data supports this notion, whereby the individual responses to the intervention displayed a wide range of variability in the TMS responses. Despite this, there are several factors known to influence the effectiveness of TDCS including, but not limited to, biological factors such as different ages, genetics, gender 67,68 and anatomical factors such as variances in individual brain anatomy such as skull thickness and cerebrospinal fluid. ${ }^{69,70}$ In addition, different electrode montages used during the application of TDCS could also influence the effect of TDCS by varying the distributed electrical field on the cerebral cortex.

The current study used anodal-high-definition-TDCS as opposed to the previous strength-training TDCS studies that used conventional TDCS.24,25,50 Generally, smaller stimulating electrodes (high-definition-TDCS) have been found to produce a more focused electrical field $33,71,72$ compared to larger-size electrodes (conventional TDCS) which induce a more dispersed electrical field across the scalp and likely stimulate cortical tissue beyond the target area. ${ }^{69,73,74}$ While high-definition-TDCS induces targeted and focused electrical fields, recent evidence has revealed that the focused current flow patterns are more susceptible to individual differences, ${ }^{75}$ thus intensifying the existing high inter-individual variability in response to TDCS. This is a notion supported by the current study.

Another important difference that should be noted is that previous studies have used different testing conditions compared to the training interventions. ${ }^{24,25}$ In fact, in both Hendy and Kidgell ${ }^{24}$ and Frazer et al. ${ }^{25}$ studies, the modulation of the corticospinal tract was measured during isometric activity, despite their training protocol being dynamic. It has been suggested that there should be a degree of task-specific testing to ensure that the task-dependent response of the corticospinal tract is similar between testing and training. ${ }^{26}$ In the current study, we specifically chose an isometric training task, so we could more precisely match the muscle actions during training and TMS testing.

\section{Limitations of the study}

There are several limitations to the present study that must not be overlooked when interpreting the results. First, we did not include an experimental condition that only involved bilateral and unilateral anodal-high-definition-TDCS, thus it remains unclear whether high-definition-TDCS alone differentially modulates ipsilateral and contralateral corticospinal excitability. This was principally not included due to Covid-19, but it should be acknowledged that previous evidence suggested that dual-site anodal-high-definitionTDCS modulates corticospinal excitability to a greater magnitude compared to unilateral anodal-TDCS. ${ }^{38}$ In addition, we only examined the cortical responses from the untrained hemisphere and not the trained hemisphere. Understanding the corticospinal responses of the trained hemisphere may have provided greater insight into the null experimental effects of the untrained hemisphere. It is possible that the corticospinal responses to strength training may have involved some level of change in the intrinsic properties of the motoneurons and changes in cortical connectivity, which we were unable to identify. Thus, 
there is a need to develop cross-education studies that utilize an integrated combination of contemporary and novel experimental approaches which include several techniques (e.g., TMS, EEG and Imaging) as these are likely to expand our current knowledge about the range and nature of the neural adaptations that are evoked by cross-education. ${ }^{76}$

\section{CONCLUSION}

In conclusion, a single session of submaximal grip training with or without anodalhigh-definition-TDCS produces no cross-education of maximal grip force nor does it affect the excitability of the ipsilateral M1.

\section{REFERENCES}

1. Hortobagyi T, Lambert NJ, Hill JP. Greater cross education following training with muscle lengthening than shortening. Med Sci Sports Exerc. 1997;29:107-112. 10.1097/00005768$199701000-00015$.

2. Manca A, Dragone D, Dvir Z, Deriu F. Cross-education of muscular strength following unilateral resistance training: a meta-analysis. Eur J Appl Physiol. 2017;117:2335-2354. 10.1007/s00421-017-3720-z.

3. Hendy AM, Lamon S. The Cross-Education Phenomenon: Brain and Beyond. Front Physiol. 2017;8:297. 10.3389/fphys.2017.00297.

4. Manca A, Hortobagyi T, Rothwell J, Deriu F. Neurophysiological adaptations in the untrained side in conjunction with cross-education of muscle strength: a systematic review and meta-analysis. Journal of applied physiology (Bethesda, Md : 1985). 2018;124:15021518. 10.1152/japplphysiol.01016.2017.

5. Goodwill AM, Pearce AJ, Kidgell DJ. Corticomotor plasticity following unilateral strength training. Muscle Nerve. 2012;46:384-393. 10.1002/mus.23316.

6. Kidgell DJ, Stokes MA, Pearce AJ. Strength training of one limb increases corticomotor excitability projecting to the contralateral homologous limb. Motor Control. 2011;15:247266.

7. Mason J, Frazer AK, Horvath DM, Pearce AJ, Avela J, Howatson G, et al. Ipsilateral corticomotor responses are confined to the homologous muscle following cross-education of muscular strength. Appl Physiol Nutr Metab. 2018;43:11-22. 10.1139/apnm-2017-0457.

8. Hortobagyi T, Richardson SP, Lomarev M, Shamim E, Meunier S, Russman H, et al. Interhemispheric plasticity in humans. Med Sci Sports Exerc. 2011;43:1188-1199. 10.1249/MSS.0b013e31820a94b8.

9. Ruddy KL, Carson RG. Neural pathways mediating cross education of motor function. Front Hum Neurosci. 2013;7:397. 10.3389/fnhum.2013.00397.

10. Gwin JT, Ferris DP. An EEG-based study of discrete isometric and isotonic human lower limb muscle contractions. J Neuroeng Rehabil. 2012;9:35. 10.1186/1743-0003-9-35. 
11. Siddique U, Rahman S, Frazer AK, Pearce AJ, Howatson G, Kidgell DJ. Determining the Sites of Neural Adaptations to Resistance Training: A Systematic Review and Metaanalysis. Sports Med. 2020. 10.1007/s40279-020-01258-z.

12. Coombs TA, Frazer AK, Horvath DM, Pearce AJ, Howatson G, Kidgell DJ. Crosseducation of wrist extensor strength is not influenced by non-dominant training in righthanders. Eur J Appl Physiol. 2016;116:1757-1769. 10.1007/s00421-016-3436-5.

13. Latella C, Kidgell DJ, Pearce AJ. Reduction in corticospinal inhibition in the trained and untrained limb following unilateral leg strength training. Eur J Appl Physiol. 2012;112:30973107. 10.1007/s00421-011-2289-1.

14. Manca A, Ginatempo F, Cabboi MP, Mercante B, Ortu E, Dragone D, et al. No evidence of neural adaptations following chronic unilateral isometric training of the intrinsic muscles of the hand: a randomized controlled study. Eur J Appl Physiol. 2016;116:1993-2005. 10.1007/s00421-016-3451-6.

15. Kidgell DJ, Frazer AK, Daly RM, Rantalainen T, Ruotsalainen I, Ahtiainen J, et al. Increased cross-education of muscle strength and reduced corticospinal inhibition following eccentric strength training. Neuroscience. 2015;300:566-575. 10.1016/j.neuroscience.2015.05.057.

16. Leung M, Rantalainen T, Teo WP, Kidgell D. The ipsilateral corticospinal responses to cross-education are dependent upon the motor-training intervention. Exp Brain Res. 2018;236:1331-1346. 10.1007/s00221-018-5224-4.

17. Hortobagyi T, Taylor JL, Petersen NT, Russell G, Gandevia SC. Changes in segmental and motor cortical output with contralateral muscle contractions and altered sensory inputs in humans. J Neurophysiol. 2003;90:2451-2459. 10.1152/jn.01001.2002.

18. Howatson G, Taylor MB, Rider P, Motawar BR, Mcnally MP, Solnik S, et al. Ipsilateral motor cortical responses to TMS during lengthening and shortening of the contralateral wrist flexors. Eur J Neurosci. 2011;33:978-990. 10.1111/j.1460-9568.2010.07567.x.

19. Zijdewind I, Butler JE, Gandevia SC, Taylor JL. The origin of activity in the biceps brachii muscle during voluntary contractions of the contralateral elbow flexor muscles. Exp Brain Res. 2006;175:526-535. 10.1007/s00221-006-0570-z.

20. Carson RG, Nelson BD, Buick AR, Carroll TJ, Kennedy NC, Cann RM. Characterizing changes in the excitability of corticospinal projections to proximal muscles of the upper limb. Brain Stimul. 2013;6:760-768. 10.1016/j.brs.2013.01.016.

21. Dettmers C, Fink GR, Lemon RN, Stephan KM, Passingham RE, Silbersweig D, et al. Relation between cerebral activity and force in the motor areas of the human brain. $J$ Neurophysiol. 1995;74:802-815. 10.1152/jn.1995.74.2.802.

22. Muellbacher W, Facchini S, Boroojerdi B, Hallett M. Changes in motor cortex excitability during ipsilateral hand muscle activation in humans. Clin Neurophysiol. 2000;111:344-349. 10.1016/s1388-2457(99)00243-6.

23. Hortobagyi T, Maffiuletti NA. Neural adaptations to electrical stimulation strength training. Eur J Appl Physiol. 2011;111:2439-2449. 10.1007/s00421-011-2012-2. 
24. Hendy AM, Kidgell DJ. Anodal-tDCS applied during unilateral strength training increases strength and corticospinal excitability in the untrained homologous muscle. Exp Brain Res. 2014;232:3243-3252. 10.1007/s00221-014-4016-8.

25. Frazer AK, Williams J, Spittle M, Kidgell DJ. Cross-education of muscular strength is facilitated by homeostatic plasticity. Eur J Appl Physiol. 2017;117:665-677. 10.1007/s00421-017-3538-8.

26. Brownstein CG, Ansdell P, Skarabot J, Frazer A, Kidgell D, Howatson G, et al. Motor cortical and corticospinal function differ during an isometric squat compared with isometric knee extension. Exp Physiol. 2018;103:1251-1263. 10.1113/EP086982.

27. Nitsche MA, Paulus W. Excitability changes induced in the human motor cortex by weak transcranial direct current stimulation. J Physiol. 2000;527 Pt 3:633-639. 10.1111/j.14697793.2000.t01-1-00633.x.

28. Nitsche MA, Paulus W. Sustained excitability elevations induced by transcranial DC motor cortex stimulation in humans. Neurology. 2001;57:1899-1901. 10.1212/wnl.57.10.1899.

29. Nitsche MA, Paulus W. Transcranial direct current stimulation--update 2011. Restor Neurol Neurosci. 2011;29:463-492. 10.3233/RNN-2011-0618.

30. Batsikadze G, Moliadze V, Paulus W, Kuo MF, Nitsche MA. Partially non-linear stimulation intensity-dependent effects of direct current stimulation on motor cortex excitability in humans. J Physiol. 2013;591:1987-2000. 10.1113/jphysiol.2012.249730.

31. Nitsche MA, Seeber A, Frommann K, Klein CC, Rochford C, Nitsche MS, et al. Modulating parameters of excitability during and after transcranial direct current stimulation of the human motor cortex. J Physiol. 2005;568:291-303. 10.1113/jphysiol.2005.092429.

32. Biabani M, Aminitehrani M, Zoghi M, Farrell M, Egan G, Jaberzadeh S. The effects of transcranial direct current stimulation on short-interval intracortical inhibition and intracortical facilitation: a systematic review and meta-analysis. Rev Neurosci. 2018;29:99114. 10.1515/revneuro-2017-0023.

33. Kuo HI, Bikson M, Datta A, Minhas P, Paulus W, Kuo MF, et al. Comparing cortical plasticity induced by conventional and high-definition $4 \times 1$ ring tDCS: a neurophysiological study. Brain Stimul. 2013;6:644-648. 10.1016/j.brs.2012.09.010.

34. Li DH, Gong HH, Xiao XZ, Wang JH. Functional MRI activation of primary and secondary motor areas in healthy subjects. Neural Regeneration Research. 2008;3:138-141.

35. Urbano A, Babiloni C, Onorati P, Babiloni F. Human cortical activity related to unilateral movements. A high resolution EEG study. Neuroreport. 1996;8:203-206. 10.1097/00001756-199612200-00041.

36. Chiou SY, Wang RY, Liao KK, Wu YT, Lu CF, Yang YR. Co-activation of primary motor cortex ipsilateral to muscles contracting in a unilateral motor task. Clin Neurophysiol. 2013;124:1353-1363. 10.1016/j.clinph.2013.02.001.

37. Fischer DB, Fried PJ, Ruffini G, Ripolles O, Salvador R, Banus J, et al. Multifocal tDCS targeting the resting state motor network increases cortical excitability beyond traditional 
tDCS targeting unilateral motor cortex. Neuroimage. 2017;157:34-44. 10.1016/j.neuroimage.2017.05.060.

38. Vaseghi B, Zoghi M, Jaberzadeh S. The effects of anodal-tDCS on corticospinal excitability enhancement and its after-effects: conventional vs. unihemispheric concurrent dual-site stimulation. Front Hum Neurosci. 2015;9:533. 10.3389/fnhum.2015.00533.

39. Oldfield RC. The assessment and analysis of handedness: The Edinburgh inventory. Neuropsychologia. 1971;9:97-113.

40. Keel JC, Smith MJ, Wassermann EM. A safety screening questionnaire for transcranial magnetic stimulation. Clin Neurophysiol. 2001;112:720. 10.1016/s1388-2457(00)00518-6.

41. Carr JC, Ye X, Stock MS, Bemben MG, Defreitas JM. The time course of cross-education during short-term isometric strength training. Eur J Appl Physiol. 2019;119:1395-1407. 10.1007/s00421-019-04130-9.

42. Palumbo A, Waage A, Hulin C, Beksac M, Zweegman S, Gay F, et al. Safety of thalidomide in newly diagnosed elderly myeloma patients: a meta-analysis of data from individual patients in six randomized trials. Haematologica. 2013;98:87-94. 10.3324/haematol.2012.067058.

43. Pixa NH, Steinberg F, Doppelmayr M. High-definition transcranial direct current stimulation to both primary motor cortices improves unimanual and bimanual dexterity. Neurosci Lett. 2017;643:84-88. 10.1016/j.neulet.2017.02.033.

44. Pixa NH, Steinberg F, Doppelmayr M. Effects of High-Definition Anodal Transcranial Direct Current Stimulation Applied Simultaneously to Both Primary Motor Cortices on Bimanual Sensorimotor Performance. Front Behav Neurosci. 2017;11:130. 10.3389/fnbeh.2017.00130.

45. Kidgell DJ, Stokes MA, Castricum TJ, Pearce AJ. Neurophysiological responses after short-term strength training of the biceps brachii muscle. J Strength Cond Res. 2010;24:3123-3132. 10.1519/JSC.0b013e3181f56794.

46. Hendy AM, Teo WP, Kidgell DJ. Anodal Transcranial Direct Current Stimulation Prolongs the Cross-education of Strength and Corticomotor Plasticity. Med Sci Sports Exerc. 2015;47:1788-1797. 10.1249/mss.0000000000000600.

47. Pearce AJ, Clark RA, Kidgell DJ. A comparison of two methods in acquiring stimulusresponse curves with transcranial magnetic stimulation. Brain Stimul. 2013;6:306-309. 10.1016/j.brs.2012.05.010.

48. Damron LA, Dearth DJ, Hoffman RL, Clark BC. Quantification of the corticospinal silent period evoked via transcranial magnetic stimulation. J Neurosci Methods. 2008;173:121128. 10.1016/j.jneumeth.2008.06.001.

49. Mason J, Frazer A, Horvath DM, Pearce AJ, Avela J, Howatson G, et al. Adaptations in corticospinal excitability and inhibition are not spatially confined to the agonist muscle following strength training. Eur J Appl Physiol. 2017;117:1359-1371. 10.1007/s00421-0173624-y. 
50. Frazer AK, Howatson G, Ahtiainen JP, Avela J, Rantalainen T, Kidgell DJ. Priming the Motor Cortex With Anodal Transcranial Direct Current Stimulation Affects the Acute Inhibitory Corticospinal Responses to Strength Training. J Strength Cond Res. 2019;33:307-317. 10.1519/JSC.0000000000002959.

51. Lee M, Hinder MR, Gandevia SC, Carroll TJ. The ipsilateral motor cortex contributes to cross-limb transfer of performance gains after ballistic motor practice. J Physiol. 2010;588:201-212. 10.1113/jphysiol.2009.183855.

52. Carroll TJ, Lee M, Hsu M, Sayde J. Unilateral practice of a ballistic movement causes bilateral increases in performance and corticospinal excitability. J Appl Physiol (1985). 2008;104:1656-1664. 10.1152/japplphysiol.01351.2007.

53. Hinder MR, Schmidt MW, Garry MI, Summers JJ. Unilateral contractions modulate interhemispheric inhibition most strongly and most adaptively in the homologous muscle of the contralateral limb. Exp Brain Res. 2010;205:423-433. 10.1007/s00221-010-2379-z.

54. Mason J, Frazer AK, Avela J, Pearce AJ, Howatson G, Kidgell DJ. Tracking the corticospinal responses to strength training. Eur J Appl Physiol. 2020;120:783-798. 10.1007/s00421-020-04316-6.

55. Heetkamp J, Hortobagyi T, Zijdewind I. Increased bilateral interactions in middle-aged subjects. Front Aging Neurosci. 2014;6:5. 10.3389/fnagi.2014.00005.

56. Kukulka CG, Clamann HP. Comparison of the recruitment and discharge properties of motor units in human brachial biceps and adductor pollicis during isometric contractions. Brain Res. 1981;219:45-55. 10.1016/0006-8993(81)90266-3.

57. Humphry AT, Lloyd-Davies EJ, Teare RJ, Williams KE, Strutton PH, Davey NJ. Specificity and functional impact of post-exercise depression of cortically evoked motor potentials in man. Eur J Appl Physiol. 2004;92:211-218. 10.1007/s00421-004-1082-9.

58. Leung M, Rantalainen T, Teo WP, Kidgell D. Motor cortex excitability is not differentially modulated following skill and strength training. Neuroscience. 2015;305:99-108. 10.1016/j.neuroscience.2015.08.007.

59. Colomer-Poveda D, Hortobagyi T, Keller M, Romero-Arenas S, Marquez G. Training intensity-dependent increases in corticospinal but not intracortical excitability after acute strength training. Scand J Med Sci Sports. 2020;30:652-661. 10.1111/sms.13608.

60. Schmidt MW, Hinder MR, Summers JJ, Garry MI. Long-lasting contralateral motor cortex excitability is increased by unilateral hand movement that triggers electrical stimulation of opposite homologous muscles. Neurorehabil Neural Repair. 2011;25:521-530. 10.1177/1545968310397202.

61. Manca A, Cabboi MP, Ortu E, Ginatempo F, Dragone D, Zarbo IR, et al. Effect of Contralateral Strength Training on Muscle Weakness in People With Multiple Sclerosis: Proof-of-Concept Case Series. Phys Ther. 2016;96:828-838. 10.2522/ptj.20150299.

62. Fang Y, Siemionow V, Sahgal V, Xiong F, Yue GH. Greater movement-related cortical potential during human eccentric versus concentric muscle contractions. J Neurophysiol. 2001;86:1764-1772. 10.1152/jn.2001.86.4.1764. 
63. Koch G, Fernandez Del Olmo M, Cheeran B, Ruge D, Schippling S, Caltagirone C, et al. Focal stimulation of the posterior parietal cortex increases the excitability of the ipsilateral motor cortex. J Neurosci. 2007;27:6815-6822. 10.1523/JNEUROSCI.0598-07.2007.

64. Wiethoff S, Hamada M, Rothwell JC. Variability in response to transcranial direct current stimulation of the motor cortex. Brain Stimul. 2014;7:468-475. 10.1016/j.brs.2014.02.003.

65. Lopez-Alonso V, Cheeran B, Rio-Rodriguez D, Fernandez-Del-Olmo M. Inter-individual variability in response to non-invasive brain stimulation paradigms. Brain Stimul. 2014;7:372-380. 10.1016/j.brs.2014.02.004.

66. Horvath JC, Vogrin SJ, Carter O, Cook MJ, Forte JD. Effects of a common transcranial direct current stimulation (tDCS) protocol on motor evoked potentials found to be highly variable within individuals over 9 testing sessions. Exp Brain Res. 2016;234:2629-2642. 10.1007/s00221-016-4667-8.

67. Krause B, Cohen Kadosh R. Not all brains are created equal: the relevance of individual differences in responsiveness to transcranial electrical stimulation. Front Syst Neurosci. 2014;8:25. 10.3389/fnsys.2014.00025.

68. Ridding MC, Ziemann U. Determinants of the induction of cortical plasticity by non-invasive brain stimulation in healthy subjects. J Physiol. 2010;588:2291-2304. 10.1113/jphysiol.2010.190314.

69. Opitz A, Yeagle E, Thielscher A, Schroeder C, Mehta AD, Milham MP. On the importance of precise electrode placement for targeted transcranial electric stimulation. Neuroimage. 2018;181:560-567. 10.1016/j.neuroimage.2018.07.027.

70. Laakso I, Tanaka S, Koyama S, De Santis V, Hirata A. Inter-subject Variability in Electric Fields of Motor Cortical tDCS. Brain Stimul. 2015;8:906-913. 10.1016/j.brs.2015.05.002.

71. Caparelli-Daquer EM, Zimmermann TJ, Mooshagian E, Parra LC, Rice JK, Datta A, et al. A pilot study on effects of $4 \times 1$ high-definition tDCS on motor cortex excitability. Conf Proc IEEE Eng Med Biol Soc. 2012;2012:735-738. 10.1109/EMBC.2012.6346036.

72. Datta A, Bansal V, Diaz J, Patel J, Reato D, Bikson M. Gyri-precise head model of transcranial direct current stimulation: improved spatial focality using a ring electrode versus conventional rectangular pad. Brain Stimul. 2009;2:201-207, 207 e201. 10.1016/j.brs.2009.03.005.

73. Nitsche MA, Doemkes S, Karakose T, Antal A, Liebetanz D, Lang N, et al. Shaping the effects of transcranial direct current stimulation of the human motor cortex. J Neurophysiol. 2007;97:3109-3117. 10.1152/jn.01312.2006.

74. Rahman S, Siddique U, Frazer A, Pearce A, Kidgell D. tDCS Anodal tDCS increases bilateral corticospinal excitability irrespective of hemispheric dominance. Journal of science and medicine. 2020;2. 10.37714/JOSAM.V2I2.40.

75. Mikkonen M, Laakso I, Tanaka S, Hirata A. Cost of focality in TDCS: Interindividual variability in electric fields. Brain Stimul. 2020;13:117-124. 10.1016/j.brs.2019.09.017.

76. Manca A, Hortobagyi T, Carroll TJ, Enoka RM, Farthing JP, Gandevia SC, et al. Contralateral Effects of Unilateral Strength and Skill Training: Modified Delphi Consensus 
to Establish Key Aspects of Cross-Education. Sports Med. 2021;51:11-20.

10.1007/s40279-020-01377-7

\section{ACKNOWLEDGEMENTS}

The Authors sincerely thank Dr Eric J. Frazer for providing proof reading and feedback on manuscript drafts.

Citation: Alibazi RJ, Frazer AK, Tallent J, Pearce AJ, Hortobágyi T, Kidgell D. (2021). A single session of submaximal grip strength training with or without high-definition anodal-TDCS produces no cross-education of maximal force. Brazilian Journal of Motor Behavior, 15(3): 216-236.

Editors: Dr Fabio Augusto Barbieri - São Paulo State University (UNESP), Bauru, SP, Brazil; Dr José Angelo Barela São Paulo State University (UNESP), Rio Claro, SP, Brazil; Dr Natalia Madalena Rinaldi - Federal University of Espírito Santo (UFES), Vitória, ES, Brazil.

Copyright:@ 2021 Alibazi, Frazer, Tallent, Pearce, Hortobágyi and Kidgell and BJMB. This is an open-access article distributed under the terms of the Creative Commons Attribution-Non Commercial-No Derivatives 4.0 International License which permits unrestricted use, distribution, and reproduction in any medium, provided the original author and source are credited.

Funding: This research did not receive any specific grant from funding agencies in the public, commercial, or not-forprofit sectors.

Competing interests: The authors have declared that no competing interests exist.

DOI: https://doi.org/10.20338/bjmb.v15i3.223 